\title{
Analytical Techniques
}

\section{XRF Major element analysis}

Samples were ground in an agate mill to avoid any possible trace element contamination. Major element analyses were performed at the School of Earth Sciences (UTAS) using X-ray fluorescence spectrometry (XRF) and the methods of Robinson et al. [2003]. Whole rock sample powders were fused with 12-22 flux (a pre-fused mixture consisting of 12 parts $\mathrm{Li}_{2} \mathrm{~B}_{4} \mathrm{O}_{7}$ and 22 parts $\mathrm{LiBO}_{2}$ ) using a sample:flux ratio of $1: 9$ at $1100^{\circ} \mathrm{C}$. All fusions are performed in a non-wetting 5\% Au- 95\% Pt alloy crucible. The following quantities are used to make $32 \mathrm{~mm}$ diameter discs: - $12-22 \mathrm{flux}(4.5000 \mathrm{~g})$, sample $(0.5000 \mathrm{~g})$ and $\mathrm{LiNO}_{3}(0.0606 \mathrm{~g}$, added as $100 \mu \mathrm{l}$ of $60.6 \% \mathrm{LiNO}_{3}$ ). The mix is fused with agitation at $1100^{\circ} \mathrm{C}$ for 15 minutes before being cast in a 5\% Au -95\% Pt mould. Ignition loss was determined on $\sim 2$ grams of sample powder ignited overnight at $1000^{\circ} \mathrm{C}$ in $5 \mathrm{ml}$ platinum crucibles. Major elements were determined with a ScMo 3kW side window X-ray tube and a Philips PW1480 x-ray spectrometer. Corrections for mass absorption are calculated using Philips X40 software with De Jongh's calibration model and Philips (or CSIRO) alpha coefficients. Compton scattering is also used for many trace elements (see below). Calibrations are on pure element oxide mixes in pure silica, along with international and Tasmanian standard rocks are used.

\section{XRF Trace element analysis}

Trace element analyses ( $\mathrm{Rb}, \mathrm{Ba}, \mathrm{Nb}, \mathrm{Sr}, \mathrm{Zr}, \mathrm{Y}, \mathrm{V}, \mathrm{Sc}, \mathrm{Cr}, \mathrm{Ni}$ ) were determined by using pressed powder pill analysis (10grams, 32mm) using a combination of ScMo (Y, Rb, Ni) and $\mathrm{Au}(\mathrm{Ba}, \mathrm{Nb}, \mathrm{Sr}, \mathrm{Zr}, \mathrm{V}, \mathrm{Sc}, \mathrm{Cr}) 3 \mathrm{~kW}$ side window X-ray tubes and a Philips PW1480 x-ray spectrometer.

\section{ICP-MS Trace element analysis}

ICP-MS trace element analyses at UTAS were obtained using the methods of Robinson et al. [1999] and Yu et al. [2000]. ICP-MS analyses were performed on duplicate high-pressure $\mathrm{HF}-\mathrm{HClO}_{4}$ digestions. Sub-boiling double distilled acids and ultra pure water were used, as were clean sampler and skimmer cones, ICP torch, spray chamber, nebuliser and sample introduction tubes (including auto-sampler tubing). Prior to sample analysis the instrument was purged for at least 24 hours with $5 \% \mathrm{v} / \mathrm{v} \mathrm{HNO}_{3}$ and $0.05 \% \mathrm{v} / \mathrm{v} \mathrm{HF}$ rinse solution.

\section{Sr-Nd-Pb isotopes}

Hand-picked rock chips were acid washed in hot $6 \mathrm{~N} \mathrm{HCl}$ for 15 minutes and then dissolved in $\mathrm{HF} / \mathrm{HNO}_{3}$ acids. After extraction of $\mathrm{Pb}$ using conventional anion exchange using $\mathrm{HBr}-\mathrm{HCl}$ media, Sr and LREE were extracted on EICHROM Sr. resin and RE.resin, respectively, followed by Nd purification on EICHROM LN resin [Pin et al., 1994; Pin and SantosZalduegui, 1997]. Total procedural blanks were negligible in all cases with $\mathrm{Pb}$ blanks typically less than $20 \mathrm{pg}$.

All isotopic analyses were carried out on a Nu Instruments multi-collector ICP-MS coupled to a CETAC Aridus desolvating nebulizer [Belshaw et al., 1998; Woodhead, 2002]. Instrumental mass bias was corrected by normalizing to ${ }^{146} \mathrm{Nd} /{ }^{145} \mathrm{Nd}=2.0719425$ (equivalent to ${ }^{146} \mathrm{Nd} /{ }^{144} \mathrm{Nd}=0.7219$, [Vance and Thirlwall, 2002] ) and ${ }^{86} \mathrm{Sr} /{ }^{88} \mathrm{Sr}=0.1194$, using the exponential law. Further details can be found in Maas et al. [2005]. In the case of MC-ICPMS data, where mass bias is generally highly constant during a given analytical session, but can 
vary between sessions, data are routinely normalised to the standards of the day. In this case, data are reported relative to La Jolla $\mathrm{Nd}=0.511860$ and SRM987 $=0.710230$. This secondary normalization yields the following results for international standards $( \pm 2 \mathrm{sd})$ : BCR-1 $=$ $0.512641 \pm 18, \mathrm{BHVO}-1=0.512998 \pm 18, \mathrm{JNdi}-1=0.512113 \pm 22 ; \mathrm{E} \& \mathrm{~A}$ Sr carbonate $=$ $0.708005 \pm 47, \mathrm{BCR}-1=0.705016 \pm 46, \mathrm{BHVO}-1=0.703478 \pm 36$. These results compare well with data based on TIMS and MC-ICPMS from other laboratories (e.g. [Raczek et al., 2003; Tanaka et al., 2000]). Typical in-run precisions (2se) are $\pm 0.000010(\mathrm{Nd})$ and \pm 0.000022 (Sr). External (2sd) precision, or reproducibility, is $\pm 0.000020(\mathrm{Nd})$ and \pm 0.000040 (Sr) and is based on the results for secondary standards. Mass bias for $\mathrm{Pb}$ was corrected using the thallium-doping technique [Woodhead, 2002]. This produces data accurate to $< \pm 0.02 \%$ (with respect to the SRM981 $\mathrm{Pb}$ standard), with an external (2sd) precision of $\approx \pm 0.02 \%$.

\section{References}

Belshaw, N.S., P.A. Freedman, R.K. O’Nions, M. Frank, and Y. Guo (1998), A new variable dispersion double-focusing plasma mass spectrometer with performance illustrated for $\mathrm{Pb}$ isotopes. International Journal of Mass Spectrometry and Ion Processes, 181, 51-58.

Maas, R., M.B. Kamenetsky, A.V. Sobolev, V. S. Kamenetsky, and N.V. Sobolev (2005), Sr$\mathrm{Nd}-\mathrm{Pb}$ isotopic evidence for a mantle origin of alkali chlorides and carbonates in the Udachnaya kimberlite, Siberia. Geology, 35, 549-552

Pin, C., D. Briot, C. Bassin, and F. Poitrasson (1994), Concomitant separation of strontium and samarium-neodymium for isotopic analysis in silicate samples, based on specific extraction chromatography. Analytica Chimica Acta, 298, 209-217.

Pin, C., and J. F. Santos-Zalduegui (1997), Sequential separation of light rare-earth elements, thorium and uranium by miniaturized extraction chromatography: application to isotopic analyses of silicate rocks. Analytica Chimica Acta, 339, 79-89.

Raczek, I., K. P. Jochum, and A. W. Hofmann (2003), Neodymium and strontium isotope data for USGS reference materials BCR-1, BCR-2, BHVO-1, BHVO-2, AGV-1, AGV-2, GSP-1, GSP-2 and eight MPI-DING reference glasses. Geostandards Newsletter, 27, $173-$ 179

Robinson, P. (2003), XRF analysis of flux-fused discs, Geoanalysis 2003, The 5th International Conference on the Analysis of Geological and Environmental Materials, Abstracts, 90.

Robinson, P., A. T. Townsend, Z. Yu, and C. Munker (1999), Determination of Scandium, Yttrium and Rare Earth Elements in rocks by high resolution inductively coupled plasmamass spectrometry, Geostandards Newsletter, 23, 31-46.

Tanaka, T., and 18 others (2000), JNdi-1: a neodymium isotopic reference in consistency with La Jolla neodymium. Chemical Geology, 168, 279-281.

Vance, D., and M. F. Thirlwall (2002), An assessment of mass discrimination in MC-ICPMS using Nd isotopes. Chemical Geology, 185, 227-240.

Woodhead, J.D. (2002), A simple method for obtaining highly accurate $\mathrm{Pb}$ isotope data by MC-ICP-MS. Journal of Analytical Atomic Spectrometry, 17, 1-6

Yu Z., P. Robinson, A. T. Townsend, C. Munker, and A. J. Crawford (2000), Determination of HFSE, $\mathrm{Rb}, \mathrm{Sr}, \mathrm{Mo}, \mathrm{Sb}, \mathrm{Cs}, \mathrm{Tl}$ and $\mathrm{Bi}$ at ng g-1 levels in geological reference materials by magnetic sector ICP-MS after HF/HCLO4 high pressure digestion, Geostandards Newsletter, 24, 39-50. 


\section{GEOLOGICAL SETTING}

The Kadavu Island Group (Figure S1) lies approximately $88 \mathrm{~km}$ south of the main Fijian Island of Viti Levu in the southwest Pacific. It forms the northernmost and emergent part of the submarine Hunter Ridge, and is separated from the main Fijian Platform (defined by an area of shallow water, generally less than $1 \mathrm{~km}$ deep), upon which the major Fiji islands are located (Colley and Hindle, 1984; Rodda, 1994) by the relatively deep water (2000-2500m) of the Kadavu Channel. The Kadavu Trench lies between Kadavu Island and seafloor of the South Fiji Basin.

The submarine Hunter Ridge forms the south-eastern boundary of the North Fiji Basin, linking the southern end of the Vanuatu Arc with the Fiji Platform. It is part of the tectonically complex New Hebrides-Fiji Orogen (Bird, 2003). The number and nature of plate boundaries in this area is poorly defined (Bird, 2003). The Hunter Ridge is a series of submarine ridges, associated in part with large sinistral earthquakes, that separates the actively spreading North Fiji Basin from the extinct South Fiji Basin. It is not clear whether it forms part of a plate boundary or not (Bird, 2003). Auzende et al. (1996) proposed that the Hunter Ridge was an embryonic island arc formed during a short period ( 7-3Ma) of northdirected subduction of the South Fiji Basin oceanic crust under the North Fiji Basin and Fiji Platform. The Kadavu Trench was formed as a result of this process. The development of this arc was aborted when northward subduction ceased due to plate reorganisation. Presently, there is no evidence for active arc volcanoes on the Hunter Ridge, apart from those occurring within an active rift at the southern end of the Hunter Ridge (Danyushevsky et al. 2006) where the main N-S orientated spreading centre of the North Fiji Basin is transecting the Hunter Ridge. Limited sampling of the Hunter Ridge to the southwest of Kadavu Island has recovered volcanics comprising a typical low-K island arc tholeiite suite (Verbeeten, 1996). 
The volcanic rocks of the Kadavu Island Group range in age from $3.4-0.36 \mathrm{Ma}$ (Figure S1, Whelan et al.,1985) and are the youngest subduction-related volcanism on the Fijian Islands. Kadavu is the only island within the Fiji archipelago where adakites have been described. Geological mapping by Woodrow (1980), and our fieldwork, demonstrates that the Kadavu Island Group is composed of a number of volcanic units forming three main volcanic groups that young from north to south. The oldest group ( 3.4 Ma, Whelan et al., 1985) are the shoshonitic lavas forming the Astrolabe Islands. The youngest groups are the adakitic lavas of Eastern and Western Kadavu ( 0.5-2.9 Ma; Whelan et al., 1985).

Shoshonitic magmatism in Fiji occurred from $\sim 5.5$ to 3.0 Ma (Whelan et al., 1985), with Astrolabe shoshonites being the youngest. The Astrolabe shoshonite lavas range from relatively primitive olivine + clinopyroxene - phyric absorakites (e.g. AV186, Table 1) to relatively more differentiated plagioclase + clinopyroxene + biotite + hornblende - phyric andesites. Their parental magmas probably represent relatively low degree partial melts of a refractory mantle source which had a previous history of enrichment by subduction zone fluids (Leslie, 2005). This source enrichment most likely took place during subduction at the paleo-Vitiaz trench. Post-subduction rifting and rotation of the Fiji Platform and surrounding areas since $5 \mathrm{Ma}$ induced decompression melting of these enriched subduction modified mantle sources (Gill et al. 1984; Leslie, 2005).

The oldest adakite lavas occur in Eastern Kadavu and the small island of Ono $(\sim 2.8$ Ma). The youngest adakite lavas occur in Western Kadavu (Figure S1) and consist of two subgroups. The first are HSA lavas, and the second are relatively primitive LSA lavas forming the small islet of Ngaloa (Figure S1) as well as isolated outcrops overlying the older adakites of Eastern Kadavu. Although the youngest age reported by Whelan et al. (1985) of 0.36 Ma is from Ngaloa Island, from the presence of magmatic enclaves of Ngaloa LSA within the Western Kadavu HSA lavas indicates that both the LSA magmas of Ngaloa and the 
HSA magmas of Western Kadavu were erupting simultaneously. The HSA adakites from both the Eastern and Western Kadavu are andesitic to dacitic in composition and contain up to 60 vol\% phenocrysts of plagioclase, clinopyroxene, hornblende, biotite and magnetite, with minor altered olivine (2-7 vol\%) present in some of the more magnesian adakites from Western Kadavu (Verbeeten, 1996).

\section{References:}

Auzende, J.M., Pelletier, B. and Eissen J.P., 1996, The North Fiji Basin: geology, structure and geodynamic evolution: In, Taylor, B (ed). Backarc basins: tectonics and magmatism, Plenum Press, New York. pp. 139-175.

Bird, P., 2003, An updated digital model of plate boundaries: Geochemistry, Geophysics, Geosystems, v. 4, no. 3, doi:10.1029/2001GC000252.

Colley, H. and Hindle, W. H., 1984, Volcano-tectonic evolution of Fiji and adjoining marginal basins. In: Kokelaar, B.P. and Howells, M.F. (eds), Marginal Basin Geology: Geological Society of London, pp 151-162.

Danyushevsky, L.V., Falloon, T.J., and Crawford, A.J., 2006, Subduction-related magmatism at the southern tip of the North Fiji backarc basin: Extended Abstracts (CD), Australian Earth Sciences Convention 2006, 2-6 July 2006, Melbourne, Australia. 1-8.

Gill, J. B., Stork, A. L., and Whelan, P. M., 1984, Volcanism accompanying backarc basin development in the southwest Pacific: Tectonophysics, v. 102, p. 207-224. 
Leslie, R.A.J., 2005, Primitive Shoshonites from Fiji: Mineralogy, Melt Inclusions and Geochemistry: Unpubl PhD thesis, UTAS, pp. 360.

Rhodda, P., 1994, Geology of Fiji. In, Stevenson, A. J., Herzer, R. H., and Balance, P. F. (eds), Geology and submarine resources of the Tonga Lau - Fiji Region. SOPAC Technical Bulletin, v. 8, p. 131-151.

Verbeeten, A., 1996, Petrology, Geochemistry and tectonic implications of magmatism along the northern Hunter Ridge and Kadavu Island group, Fiji: Unpubl PhD thesis, UTAS, pp. 190.

Whelan, P.M., Gill, J. B., Kollman, E., Duncan, R. A. \& Drake, R. E., 1985, Radiometric dating of magmatic stages in Fiji: In: Scholl, D.W. \& Vallier, T.L. (eds), Geology and offshore resources of Pacific island arcs - Tonga region. Circum-Pacific Council for Energy and Mineral Resources Earth Science Series, v.2. Houston, Texas.

Woodrow, P.J., 1980, Geology of Kadavu: Mineral Resources Department, Fiji, Bulletin no. 7. 


\begin{tabular}{|c|c|c|c|}
\hline Sample № & Texture & Phenocrysts and microphenocrysts & Groundmass \\
\hline \multicolumn{4}{|c|}{ WESTERN KADAVU } \\
\hline ST1 & $\begin{array}{c}\text { highly } \\
\text { porphyritic }\end{array}$ & $\begin{array}{l}\text { plagioclase: } 35 \%, 0.5-5 \mathrm{~mm} \text { (some grains up to } 12 \mathrm{~mm} \text { ), } \\
\text { subhedral-euhedral, oscillatory zoned. } \\
\text { hornblende: } 5 \%, 0.3-0.6 \mathrm{~mm} \text {, subhedral, opacitic rims. } \\
\text { biotite: } 2 \%, 0.2-0.9 \mathrm{~mm} \text {, subhedral, opacitic rims. } \\
\text { magnetite: } 2 \%, 0.05-0.2 \mathrm{~mm} \text {. }\end{array}$ & $\begin{array}{l}\text { fine grained to glassy consisting } \\
\text { of plagioclase microlites and } \\
\text { magnetite. }\end{array}$ \\
\hline ST5 & $\begin{array}{c}\text { highly } \\
\text { porphyritic }\end{array}$ & $\begin{array}{l}\text { plagioclase: } 35 \%, 0.5-4 \mathrm{~mm} \text { (some grains up to } 10 \mathrm{~mm} \text { ), } \\
\text { subhedral, oscillatory zoned. } \\
\text { hornblende: } 15 \%, 0.2-0.9 \mathrm{~mm} \text { (some grains up to } 4 \mathrm{~mm} \text { ), } \\
\text { subhedral-euhedral, partially to totally replaced with } \\
\text { magnetite. } \\
\text { biotite: } 7 \%, 0.4-2 \mathrm{~mm} \text {, subhedral, partially to totally replaced } \\
\text { with magnetite. } \\
\text { clinopyroxene: } 3 \%, 0.2-0.6 \mathrm{~mm} \text {, subhedral-anhedral, } \\
\text { sometimes in crystal clusters. } \\
\text { magnetite: } 1 \%, 0.05-0.1 \mathrm{~mm} \text {. }\end{array}$ & $\begin{array}{l}\text { glassy containing plagioclase } \\
\text { laths and magnetite, glass } \\
\text { partially recrystallised. }\end{array}$ \\
\hline ST14 & $\begin{array}{c}\text { highly } \\
\text { porphyritic }\end{array}$ & $\begin{array}{l}\text { plagioclase: } 20 \%, 0.8-4 \mathrm{~mm} \text {, subhedral-euhedral, oscillatory } \\
\text { zoned. } \\
\text { biotite: } 10 \%, 0.5-5 \mathrm{~mm} \text {, subhedral. } \\
\text { hornblende: } 7 \%, 0.2-1.5 \mathrm{~mm} \text {, subhedral-anhedral. } \\
\text { magnetite: } 3 \%, 0.1-0.2 \mathrm{~mm} \text {. }\end{array}$ & $\begin{array}{l}\text { fine grained consisting of } \\
\text { plagioclase microlites, magnetite } \\
\text { and minor hornblende. }\end{array}$ \\
\hline ST16 & $\begin{array}{c}\text { highly } \\
\text { porphyritic }\end{array}$ & $\begin{array}{l}\text { plagioclase: } 60 \%, 0.3-2 \mathrm{~mm} \text {, subhedral-euhedral, zoned, } \\
\text { sieve like texture } \\
\text { clinopyroxene: } 7 \%, 0.1-0.6 \mathrm{~mm} \text {, subhedral-anhedral, } \\
\text { microphenocrysts only. } \\
\text { magnetite: } 3 \%, 0.05-0.2 \mathrm{~mm} \text {. }\end{array}$ & $\begin{array}{l}\text { fine grained consisting of } \\
\text { plagioclase needles and } \\
\text { magnetite. }\end{array}$ \\
\hline ST17 & $\begin{array}{c}\text { highly } \\
\text { porphyritic }\end{array}$ & $\begin{array}{l}\text { plagioclase: } 60 \%, 0.3-2 \mathrm{~mm} \text {, subhedral-euhedral, strongly } \\
\text { zoned, sieve like texture. } \\
\text { clinopyroxene: } 10 \%, 0.1-0.6 \mathrm{~mm} \text {, light-green, subhedral, } \\
\text { sometimes in clusters with hornblende and magnetite. }\end{array}$ & $\begin{array}{l}\text { fine grained consisting of } \\
\text { plagioclase microlites and } \\
\text { magnetite. }\end{array}$ \\
\hline
\end{tabular}




\begin{tabular}{|c|c|c|c|}
\hline & & $\begin{array}{l}\text { hornblende: } 1 \%, 0.1-0.4 \mathrm{~mm} \text {, subhedral, rarely zoned. } \\
\text { magnetite: } 2 \%, 0.05-0.2 \mathrm{~mm} \text {. }\end{array}$ & \\
\hline ST18 & porphyritic & $\begin{array}{l}\text { plagioclase: } 50 \%, 0.3-2 \mathrm{~mm} \text {, subhedral, oscillatory zoned. } \\
\text { clinopyroxene: } 10 \%, 0.1-0.6 \mathrm{~mm} \text {, subhedral-anhedral, } \\
\text { contains inclusions of magnetite. } \\
\text { hornblende: }<1 \%, 0.1-0.4 \mathrm{~mm} \text {, subhedral, opacitic rims, } \\
\text { contains inclusions of magnetite. } \\
\text { magnetite: } 3 \%, 0.05-0.1 \mathrm{~mm} \text {. }\end{array}$ & $\begin{array}{l}\text { fine grained consisting of } \\
\text { plagioclase laths, magnetite and } \\
\text { rare clinopyroxene. }\end{array}$ \\
\hline ST19 & $\begin{array}{c}\text { highly } \\
\text { porphyritic }\end{array}$ & $\begin{array}{l}\text { plagioclase: } 50 \%, 0.3-2 \mathrm{~mm} \text {, subhedral-euhedral, strongly } \\
\text { zoned, sieve like texture. } \\
\text { clinopyroxene: } 7 \%, 0.1-1.3 \mathrm{~mm} \text {, light-green, subhedral } \\
\text { contains inclusions of magnetite. } \\
\text { hornblende: } 1 \%, 0.1-0.4 \mathrm{~mm} \text {, subhedral-anhedral, opacitic } \\
\text { rims, contains inclusions of magnetite. } \\
\text { magnetite: } 3 \%, 0.05-0.1 \mathrm{~mm} \text {. }\end{array}$ & $\begin{array}{l}\text { fine grained consisting of } \\
\text { plagioclase laths, magnetite and } \\
\text { rare clinopyroxene. }\end{array}$ \\
\hline ST20 & porphyritic & $\begin{array}{l}\text { plagioclase: } 30 \%, 0.4-3 \mathrm{~mm} \text {, subhedral-anhedral, zoned. } \\
\text { hornblende: } 15 \%, 0.1-1 \mathrm{~mm} \text {, subhedral, partially to totally } \\
\text { replaced with magnetite (+hematite?). } \\
\text { biotite: } 10 \%, 0.2-1 \mathrm{~mm} \text {, subhedral, partially to totally } \\
\text { replaced with magnetite (+hematite?) and chlorite. } \\
\text { clinopyroxene: } 4 \%, 0.05-0.3 \mathrm{~mm} \text {, microphenocrysts only, } \\
\text { anhedral, mostly in crystal clusters, some grains partially } \\
\text { resorbed. } \\
\text { magnetite: } 2 \%, 0.05-0.1 \mathrm{~mm} \text {. }\end{array}$ & $\begin{array}{l}\text { grained consisting of plagioclase } \\
\text { microlites and magnetite. }\end{array}$ \\
\hline ST21 & porphyritic & $\begin{array}{l}\text { plagioclase: } 25 \%, 0.3-2 \mathrm{~mm} \text {, subhedral, oscillatory zoned. } \\
\text { clinopyroxene: } 5 \%, 0.1-0.5 \mathrm{~mm} \text {, anhedral, contains } \\
\text { inclusions of magnetite. } \\
\text { hornblende: }<1 \%, 0.1-0.4 \mathrm{~mm} \text {, subhedral-anhedral, few } \\
\text { singular grains. } \\
\text { magnetite: } 3 \%, 0.05-0.1 \mathrm{~mm} \text {. }\end{array}$ & $\begin{array}{l}\text { glassy containing plagioclase } \\
\text { needles and magnetite, glass } \\
\text { partially recrystallised, altered. }\end{array}$ \\
\hline ST22 & highly & plagioclase: $50 \%, 0.2-1 \mathrm{~mm}$, subhedral, oscillatory zoned. & glassy containing plagioclase \\
\hline
\end{tabular}




\begin{tabular}{|c|c|c|c|}
\hline & porphyritic & $\begin{array}{l}\text { clinopyroxene: } 7 \%, 0.1-0.8 \mathrm{~mm} \text {, subhedral-anhedral, } \\
\text { inclusions of magnetite. } \\
\text { magnetite: } 3 \%, 0.05-0.1 \mathrm{~mm} \text {. }\end{array}$ & $\begin{array}{l}\text { laths and magnetite, glass } \\
\text { recrystallised, altered. }\end{array}$ \\
\hline ST23 & $\begin{array}{l}\text { porphyritic, } \\
\text { vesicular }\end{array}$ & $\begin{array}{l}\text { plagioclase: } 20 \%, 0.3-1 \mathrm{~mm} \text {, subhedral-euhedral, strongly } \\
\text { zoned. } \\
\text { clinopyroxene: } 10 \%, 0.2-1 \mathrm{~mm} \text {, subhedral-anhedral, contains } \\
\text { inclusions of magnetite, partially resorbed. } \\
\text { magnetite: } 3 \%, 0.05-0.1 \mathrm{~mm} \text {. }\end{array}$ & $\begin{array}{l}\text { fine grained to glassy consisting } \\
\text { of plagioclase laths and } \\
\text { magnetite, glass recrystallised, } \\
\text { altered. }\end{array}$ \\
\hline ST24 & $\begin{array}{l}\text { porphyritic, } \\
\text { vesicular }\end{array}$ & $\begin{array}{l}\text { plagioclase: } 25 \%, 0.1-0.8 \mathrm{~mm} \text {, subhedral, partially altered. } \\
\text { clinopyroxene: } 7 \%, 0.1-0.8 \mathrm{~mm} \text {, anhedral, partially altered. } \\
\text { magnetite: } 3 \%, 0.05-0.1 \mathrm{~mm} \text {. }\end{array}$ & $\begin{array}{l}\text { fine grained to glassy consisting } \\
\text { of plagioclase microlites and } \\
\text { magnetite, altered. }\end{array}$ \\
\hline ST25 & $\begin{array}{c}\text { highly } \\
\text { porphyritic }\end{array}$ & $\begin{array}{l}\text { plagioclase: } 50 \%, 0.2-1.5 \mathrm{~mm} \text {, subhedral-euhedral, } \\
\text { oscillatory zoned. } \\
\text { clinopyroxene: } 10 \%, 0.1-1 \mathrm{~mm} \text {, subhedral-anhedral, contains } \\
\text { inclusions of magnetite, some grains partially replaced with } \\
\text { magnetite. } \\
\text { magnetite: } 3 \%, 0.05-0.1 \mathrm{~mm} \text {. }\end{array}$ & $\begin{array}{l}\text { fine grained consisting of } \\
\text { plagioclase microlites and } \\
\text { magnetite. }\end{array}$ \\
\hline ST26 & porphyritic & $\begin{array}{l}\text { plagioclase: } 15 \%, 0.2-1 \mathrm{~mm} \text {, subhedral, strongly zoned, } \\
\text { sieve like texture, inclusions of clinopyroxene. } \\
\text { clinopyroxene: } 20 \%, 0.1-1.5 \mathrm{~mm} \text {, subhedral-anhedral, zoned. } \\
\text { olivine: } 2 \%, 0.1-0.5 \mathrm{~mm} \text {, completely altered. } \\
\text { magnetite: } 2 \%, 0.05-0.1 \mathrm{~mm} \text {. }\end{array}$ & $\begin{array}{l}\text { fine grained to glassy consisting } \\
\text { of plagioclase laths, magnetite } \\
\text { and rare clinopyroxene, glass } \\
\text { recrystallised. }\end{array}$ \\
\hline ST27 & porphyritic & $\begin{array}{l}\text { plagioclase: } 15 \%, 0.2-1.5 \mathrm{~mm} \text {, subhedral, oscillatory zoned, } \\
\text { sieve like texture. } \\
\text { clinopyroxene: } 15 \%, 0.1-1.5 \mathrm{~mm} \text {, subhedral-anhedral, zoned, } \\
\text { some cores altered. } \\
\text { olivine: } 5 \%, 0.1-0.6 \mathrm{~mm} \text {, completely altered. } \\
\text { magnetite: } 2 \%, 0.05-0.2 \mathrm{~mm} \text {. }\end{array}$ & $\begin{array}{l}\text { grained consisting of plagioclase } \\
\text { microlites magnetite and } \\
\text { clinopyroxene, altered. }\end{array}$ \\
\hline ST28 & $\begin{array}{l}\text { highly } \\
\text { porphyritic }\end{array}$ & $\begin{array}{l}\text { plagioclase: } 35 \%, 0.3-2 \mathrm{~mm} \text {, subhedral, zoned. } \\
\text { clinopyroxene: } 15 \%, 0.1-1.5 \mathrm{~mm} \text {, subhedral-euhedral, } \\
\text { contains inclusions of magnetite. }\end{array}$ & $\begin{array}{l}\text { grained consisting of plagioclase } \\
\text { laths, magnetite and rare } \\
\text { clinopyroxene. }\end{array}$ \\
\hline
\end{tabular}




\begin{tabular}{|c|c|c|c|}
\hline & & $\begin{array}{l}\text { hornblende: } 5 \%, 0.4-1.5 \mathrm{~mm} \text {, subhedral, partially replaced } \\
\text { with magnetite. } \\
\text { magnetite: } 3 \%, 0.05-0.1 \mathrm{~mm} \text {. }\end{array}$ & \\
\hline ST29 & $\begin{array}{l}\text { highly } \\
\text { porphyritic }\end{array}$ & $\begin{array}{l}\text { plagioclase: } 50 \%, 0.2-2 \mathrm{~mm} \text {, subhedral-euhedral, strongly } \\
\text { zoned, sieve like texture. } \\
\text { clinopyroxene: } 7 \%, 0.1-0.9 \mathrm{~mm} \text {, subhedral-euhedral, } \\
\text { contains inclusions of magnetite. } \\
\text { magnetite: } 4 \%, 0.05-0.1 \mathrm{~mm} \text {. }\end{array}$ & $\begin{array}{l}\text { fine grained consisting of } \\
\text { plagioclase laths and magnetite. }\end{array}$ \\
\hline ST31 & porphyritic & $\begin{array}{l}\text { plagioclase: } 15 \%, 0.2-1.5 \mathrm{~mm} \text {, subhedral, oscillatory zoned. } \\
\text { clinopyroxene: } 15 \%, 0.1-1.5 \mathrm{~mm} \text {, subhedral, zoned, sieve } \\
\text { like texture. } \\
\text { olivine: } 7 \%, 0.1-0.6 \mathrm{~mm} \text {, completely altered. } \\
\text { magnetite: } 2 \%, 0.05-0.2 \mathrm{~mm} \text {. }\end{array}$ & $\begin{array}{l}\text { grained consisting of plagioclase } \\
\text { microlites magnetite and } \\
\text { clinopyroxene, altered. }\end{array}$ \\
\hline \multicolumn{4}{|c|}{ EASTERN KADAVU } \\
\hline ST43 & porphyritic & $\begin{array}{l}\text { plagioclase: }<1 \%, 0.1-0.3 \mathrm{~mm} \text {, microphenocrysts only, } \\
\text { subhedral. } \\
\text { clinopyroxene: } 10 \%, 0.1-1.5 \mathrm{~mm} \text {, subhedral, sometimes in } \\
\text { clusters with olivine, two types - colourless, slightly altered, } \\
\text { some cores with sieve like texture; grains with small bottle- } \\
\text { green cores and colourless rims. } \\
\text { olivine: } 5 \%, 0.1-0.6 \mathrm{~mm} \text {, subhedral, resorbed, slightly } \\
\text { altered. } \\
\text { magnetite: } 3 \%, 0.05-0.1 \mathrm{~mm} \text {. }\end{array}$ & $\begin{array}{l}\text { grained consisting of plagioclase } \\
\text { laths, clinopyroxene and } \\
\text { magnetite. }\end{array}$ \\
\hline ST44 & porphyritic & $\begin{array}{l}\text { plagioclase: }<1 \%, 0.1-0.3 \mathrm{~mm} \text {, microphenocrysts only, } \\
\text { subhedral. } \\
\text { clinopyroxene: } 10 \%, 0.1-1.2 \mathrm{~mm} \text {, subhedral, commonly in } \\
\text { clusters with olivine, two types - colourless, slightly altered, } \\
\text { slightly resorbed, some cores with sieve like texture; grains } \\
\text { with small bottle-green cores and colourless rims. } \\
\text { olivine: } 5 \%, 0.1-0.8 \mathrm{~mm} \text {, subhedral, slightly altered. } \\
\text { magnetite: } 3 \%, 0.05-0.1 \mathrm{~mm} \text {. }\end{array}$ & $\begin{array}{l}\text { grained consisting of plagioclase } \\
\text { laths, clinopyroxene and } \\
\text { magnetite. }\end{array}$ \\
\hline
\end{tabular}




\begin{tabular}{|c|c|c|c|}
\hline \multicolumn{4}{|c|}{ NGALOA } \\
\hline ST45 & $\begin{array}{l}\text { porphyritic, } \\
\text { highly } \\
\text { oxidised }\end{array}$ & $\begin{array}{l}\text { clinopyroxene: } 10 \%, 0.1-1.3 \mathrm{~mm} \text {, subhedral, three types - } \\
\text { colourless, slightly altered; cores with sieve like texture, } \\
\text { slightly resorbed; light- to bottle-green cores with colourless } \\
\text { rims. } \\
\text { olivine: } 3 \%, 0.1-0.3 \mathrm{~mm} \text {, microphenocrysts only, subhedral, } \\
\text { totally replaced with magnetite and iddingsite. } \\
\text { magnetite: } 3 \%, 0.05-0.1 \mathrm{~mm} \text {. }\end{array}$ & $\begin{array}{l}\text { fine grained consisting of } \\
\text { plagioclase laths, clinopyroxene } \\
\text { and magnetite, altered (oxidised). }\end{array}$ \\
\hline ST46 & $\begin{array}{l}\text { porphyritic, } \\
\text { highly } \\
\text { oxidised }\end{array}$ & $\begin{array}{l}\text { plagioclase: }<1 \%, 0.1-0.4 \mathrm{~mm} \text {, subhedral, strongly zoned. } \\
\text { clinopyroxene: } 7 \%, 0.1-0.9 \mathrm{~mm} \text {, subhedral, three types - } \\
\text { colourless; cores with sieve like texture, slightly resorbed; } \\
\text { bottle-green cores with colourless rims. } \\
\text { olivine: } 2 \%, 0.1-0.2 \mathrm{~mm} \text {, microphenocrysts only, subhedral- } \\
\text { anhedral, partially altered to iddingsite. } \\
\text { magnetite: } 3 \%, 0.05-0.1 \mathrm{~mm} \text {. }\end{array}$ & $\begin{array}{l}\text { fine grained consisting of } \\
\text { plagioclase laths, clinopyroxene, } \\
\text { magnetite and rare phlogopite } \\
\text { needles, altered (oxidised). }\end{array}$ \\
\hline ST47 & $\begin{array}{l}\text { porphyritic, } \\
\text { highly } \\
\text { oxidised }\end{array}$ & $\begin{array}{l}\text { clinopyroxene: } 10 \%, 0.1-0.9 \mathrm{~mm} \text {, subhedral, sometimes in } \\
\text { crystal clusters, three types - colourless; cores with sieve } \\
\text { like texture, slightly resorbed; bottle-green cores with } \\
\text { colourless rims. } \\
\text { olivine: } 1 \%, 0.1-0.2 \mathrm{~mm} \text {, microphenocrysts only, subhedral- } \\
\text { anhedral, magnetite rims, totally replaced with magnetite } \\
\text { and iddingsite. } \\
\text { magnetite: } 5 \%, 0.05-0.1 \mathrm{~mm} \text {. }\end{array}$ & $\begin{array}{l}\text { fine grained consisting of } \\
\text { plagioclase laths, clinopyroxene, } \\
\text { magnetite and rare phlogopite } \\
\text { needles, altered (oxidised). }\end{array}$ \\
\hline ST48 & $\begin{array}{l}\text { porphyritic, } \\
\text { highly } \\
\text { oxidised }\end{array}$ & $\begin{array}{l}\text { clinopyroxene: } 15 \%, 0.2-1.5 \mathrm{~mm} \text {, subhedral, three types - } \\
\text { colourless, slightly altered; cores with sieve like texture, } \\
\text { slightly resorbed; bottle-green cores (containing inclusions } \\
\text { of magnetite) with colourless rims. } \\
\text { olivine: } 3 \%, 0.1-0.6 \mathrm{~mm} \text {, subhedral, slightly resorbed, } \\
\text { inclusions of magnetite, slightly replaced with magnetite. } \\
\text { magnetite: } 5 \%, 0.05-0.1 \mathrm{~mm} \text {. }\end{array}$ & $\begin{array}{l}\text { fine grained consisting of } \\
\text { plagioclase laths, clinopyroxene } \\
\text { and magnetite, altered (highly } \\
\text { oxidised). }\end{array}$ \\
\hline ST49 & porphyritic, & plagioclase: $<1 \%, 0.1-0.3 \mathrm{~mm}$, microphenocrysts only, & grained consisting of plagioclase \\
\hline
\end{tabular}




\begin{tabular}{|c|c|c|c|}
\hline & $\begin{array}{c}\text { highly } \\
\text { oxidised }\end{array}$ & $\begin{array}{l}\text { subhedral, zoned. } \\
\text { clinopyroxene: } 15 \%, 0.2-1.8 \mathrm{~mm} \text {, subhedral, some cores with } \\
\text { sieve like texture, commonly in crystal aggregates, slightly } \\
\text { resorbed. } \\
\text { olivine: } 1 \%, 0.1-0.2 \mathrm{~mm} \text {, microphenocrysts only, subhedral- } \\
\text { anhedral, magnetite rims, slightly resorbed, slightly replaced } \\
\text { with magnetite. } \\
\text { magnetite: } 5 \%, 0.05-0.1 \mathrm{~mm} \text {. }\end{array}$ & $\begin{array}{l}\text { laths, clinopyroxene and } \\
\text { magnetite, altered (highly } \\
\text { oxidised). }\end{array}$ \\
\hline NG1 & $\begin{array}{c}\text { porphyritic, } \\
\text { oxidised }\end{array}$ & $\begin{array}{l}\text { clinopyroxene: } 10 \%, 0.2-2 \mathrm{~mm} \text {, subhedral, commonly in } \\
\text { clusters with olivine and phlogopite, two types - colourless, } \\
\text { cores usually with sieve like texture; rare grains with small } \\
\text { bottle-green cores and colourless rims. } \\
\text { olivine: } 5 \%, 0.1-1 \mathrm{~mm} \text {, subhedral, slightly resorbed, slightly } \\
\text { altered. } \\
\text { phlogopite: } 3 \%, 0.1-0.4 \mathrm{~mm} \text {, anhedral, contains inclusions of } \\
\text { magnetite, commonly in clusters with olivine and } \\
\text { clinopyroxene. } \\
\text { magnetite: } 5 \%, 0.05-0.1 \mathrm{~mm} \text {. }\end{array}$ & $\begin{array}{l}\text { grained consisting of plagioclase } \\
\text { laths, clinopyroxene, magnetite } \\
\text { and rare phlogopite, altered } \\
\text { (highly oxidised). }\end{array}$ \\
\hline NG2 & $\begin{array}{l}\text { porphyritic, } \\
\text { oxidised }\end{array}$ & $\begin{array}{l}\text { clinopyroxene: } 10 \%, 0.1-0.8 \mathrm{~mm} \text {, subhedral, zoned, some } \\
\text { cores with sieve like texture, slightly resorbed, commonly in } \\
\text { aggregates with olivine. } \\
\text { olivine: } 3 \%, 0.1-0.6 \mathrm{~mm} \text {, subhedral, slightly resorbed, altered } \\
\text { to iddingsite with magnetite. } \\
\text { magnetite: } 5 \%, 0.05-0.1 \mathrm{~mm} \text {. }\end{array}$ & $\begin{array}{l}\text { grained consisting of plagioclase } \\
\text { laths, clinopyroxene and } \\
\text { magnetite, altered (oxidised). }\end{array}$ \\
\hline NG3 & $\begin{array}{l}\text { porphyritic, } \\
\text { slightly } \\
\text { oxidised }\end{array}$ & $\begin{array}{l}\text { clinopyroxene: } 10 \%, 0.2-1.3 \mathrm{~mm} \text {, subhedral, most cores with } \\
\text { sieve like texture, slightly resorbed, commonly in clusters } \\
\text { with olivine and phlogopite. } \\
\text { olivine: } 5 \%, 0.1-0.6 \mathrm{~mm} \text {, subhedral, slightly resorbed, } \\
\text { magnetite rims, slightly altered. } \\
\text { phlogopite: } 2 \%, 0.1-0.2 \mathrm{~mm} \text {, anhedral, contains inclusions of } \\
\text { magnetite, microphenocrysts only, commonly in clusters }\end{array}$ & $\begin{array}{l}\text { grained consisting of plagioclase } \\
\text { laths, clinopyroxene, magnetite } \\
\text { and rare phlogopite, altered } \\
\text { (slightly oxidised). }\end{array}$ \\
\hline
\end{tabular}




\begin{tabular}{|c|c|c|c|}
\hline & & $\begin{array}{l}\text { with olivine and clinopyroxene. } \\
\text { magnetite: } 5 \%, 0.05-0.1 \mathrm{~mm} \text {. }\end{array}$ & \\
\hline NG4 & $\begin{array}{l}\text { porphyritic, } \\
\text { oxidised }\end{array}$ & $\begin{array}{l}\text { clinopyroxene: } 15 \%, 0.2-1.3 \mathrm{~mm} \text {, subhedral, commonly in } \\
\text { clusters with olivine and phlogopite, two types - colourless, } \\
\text { cores usually with sieve like texture; rare grains with small } \\
\text { bottle-green cores and colourless rims. } \\
\text { olivine: } 1 \%, 0.1-0.3 \mathrm{~mm} \text {, microphenocrysts only, subhedral- } \\
\text { anhedral, inclusions of magnetite, slightly altered. } \\
\text { phlogopite: } 2 \%, 0.1-0.2 \mathrm{~mm} \text {, anhedral, contains inclusions of } \\
\text { magnetite, microphenocrysts only, commonly in clusters } \\
\text { with olivine and clinopyroxene. } \\
\text { magnetite: } 5 \%, 0.05-0.1 \mathrm{~mm} \text {. }\end{array}$ & $\begin{array}{l}\text { grained consisting of plagioclase } \\
\text { laths, clinopyroxene and } \\
\text { magnetite, altered (oxidised). }\end{array}$ \\
\hline
\end{tabular}




\section{SUPPLEMENTARY DISCUSSION}

Alternative possibilities for the high-SiO 2 rich end member on Kadavu, which we consider less likely, include a 'normal' evolved arc magma; high-pressure crystal fractionation of a 'normal' arc magma; and melting of the lower crust under the volcano.

Figure 3c and Figure S3 (supplementary materials) the Hunter Ridge island arc series volcanics have a restricted range of low $\mathrm{Sr} / \mathrm{Y}(<25)$ and $\mathrm{La} / \mathrm{Yb}(<2)$ similar to other well known island arc tholeiite series rocks such as the Tofua Arc, Tonga. Figure S3 (supplementary materials) demonstrates that crystal fractionation of a typical island arc tholeiite series basalt such as the Hunter ridge or Tongan islands produces magmas with a wide range of $\mathrm{SiO}_{2}(\sim 50-74$ wt\%) with slowly decreasing $\mathrm{Sr} / \mathrm{Y}$ ratios ( $\sim 25$ decreasing to $<10$ with $\mathrm{SiO}_{2}$, see Figure S3). Mixing of an enriched high-Mg adakite such as the East Kadavu end-member, combined with crystal fractionation, could potentially produce a wide range of magmas with elevated Sr/Y similar to the Kadavu adakites. However, this model cannot reproduce other important features of the low-Mg Kadavu adakites, such as high $\mathrm{La} / \mathrm{Yb}$ values (Figure 3c). In contrast, mixing with slab melts can explain the observed range of Kadavu adakite compositions.

High-pressure fractionation of normal arc magmas has recently been suggested as a general model for adakite genesis (Macpherson et al., 2006). Although this model can explain the trace element features of adakites, it is not consistent with the observed order of crystallisation of calc-alkaline and tholeiitic series at high pressure. Experimental studies (Müntener et al., 2001; Green, 1992) and detailed petrology of calc-alkaline suites where there is clear evidence for high-pressure fractionation (Day et al., 1992) demonstrate that garnet is a late fractionating phase, and thus can only produce modest increases in $\mathrm{Sr} / \mathrm{Y}$ ratio. For example, the Miocene calc-alkaline suite from Northland, New Zealand (Day et al., 1992) 
ranges from basaltic andesite with $\mathrm{Sr} / \mathrm{Y}$ of $\sim 5$ to rhyolites with $\mathrm{Sr} / \mathrm{Y} \sim 50$ (see Figure S3, supplementary data). The crystallization sequence involves extensive olivine fractionation from a hydrous arc basalt before fractionation involving clinopyroxene, orthopyroxene and amphibole, leading eventually to plagioclase and garnet becoming fractionating phases. This sequence is similar to those obtained in high-pressure experimental studies (Müntener et al., 2001; Green, 1992) and results in CIPW corundum normative liquids at $\sim 60 \mathrm{wt} \% \mathrm{SiO}_{2}$ and the presence of garnet phenocrysts in dacites and rhyolites (Day et al., 1992). The Kadavu adakites are not CIPW corundum normative, they lack garnet phenocrysts and have significantly higher Sr/Y ratios than can be achieved by high-pressure crystal fractionation of normal arc basalts (see Figure S3, supplementary data).

As the Kadavu adakite magma series is erupted onto a basement formed by Hunter Ridge arc crust (Holmes et al., 1985), it is conceivable that the $\mathrm{SiO}_{2}$ rich end-member is a melt from lower crustal cumulates. We consider that this possibility is less likely based on studies of oceanic gabbros (Nonnotte et al., 2005; Koepke et al., 2007) which demonstrate that oceanic crustal melts do not have adakite compositions.

\section{References}

Day, R. A., Green, T. H., and Smith, I. E. M., 1992, The origin and significance of garnet phenocrysts and garnet-bearing xenoliths in Miocene calc-alkaline volcanics from Northland, New Zealand: Journal of Petrology, v. 33, p. 125-161.

Green, T. H., 1992, Experimental phase equilibrium studies of garnet-bearing I-type volcanics and high-level intrusives from Northland, New Zealand: Transactions of the Royal Society of Edinburgh: Earth Sciences, v. 83, p. 429-438. 
Holmes, R., Prasad, A., Honza, E., 1985, Seismic profiles from the Fiji area: In, Honza, E., Lewis, K.B., and Shipboard Party, A Marine Geological and Geophysical Survey of the Northern Tonga Ridge and Adjacent Lau Basin, Ministry of Lands, Survey and Natural Resources, Kingdom of Tonga, Field Report no.1, p. 37-41.

Koepke, J., Berndt, J., Feig, S. T., and Holtz, F., 2007, The formation of SiO2-rich melts within the deep oceanic crust by hydrous partial melting of gabbros: Contributions to Mineralogy and Petrology, v. 153, p. 67-84, doi: 10.1007/s00410-006-0135-y.

Macpherson, C.G., Dreher, S.T., and Thirlwall, M.F., 2006, Adakites without slab melting: High presure differentiation of island arc magma, Mindanao, the Philippines: Earth and Planetary Science Letters, v. 243, p. 581-593, doi: 10.1016/j.epsl.2005.12.034.

Müntener, O., Kelemen, P. B., and Grove, T. L., 2001, The role of $\mathrm{H}_{2} \mathrm{O}$ during crystallization of primitive arc magmas under uppermost mantle conditions and genesis of igneous pyroxenites: an experimental study: Contributions to Mineralogy and Petrology, v. 141, p. 643-658.

Nonnotte, P., Ceuleneer, G., and Benoit, M., 2005, Genesis of andesitic-boninitic magmas at mid-ocean ridges by melting of hydrated peridotites: Geochemical evidence from DSDP Site 334 gabbronorites: Earth and Planetary Science Letters, v. 236, p. 632-653. 


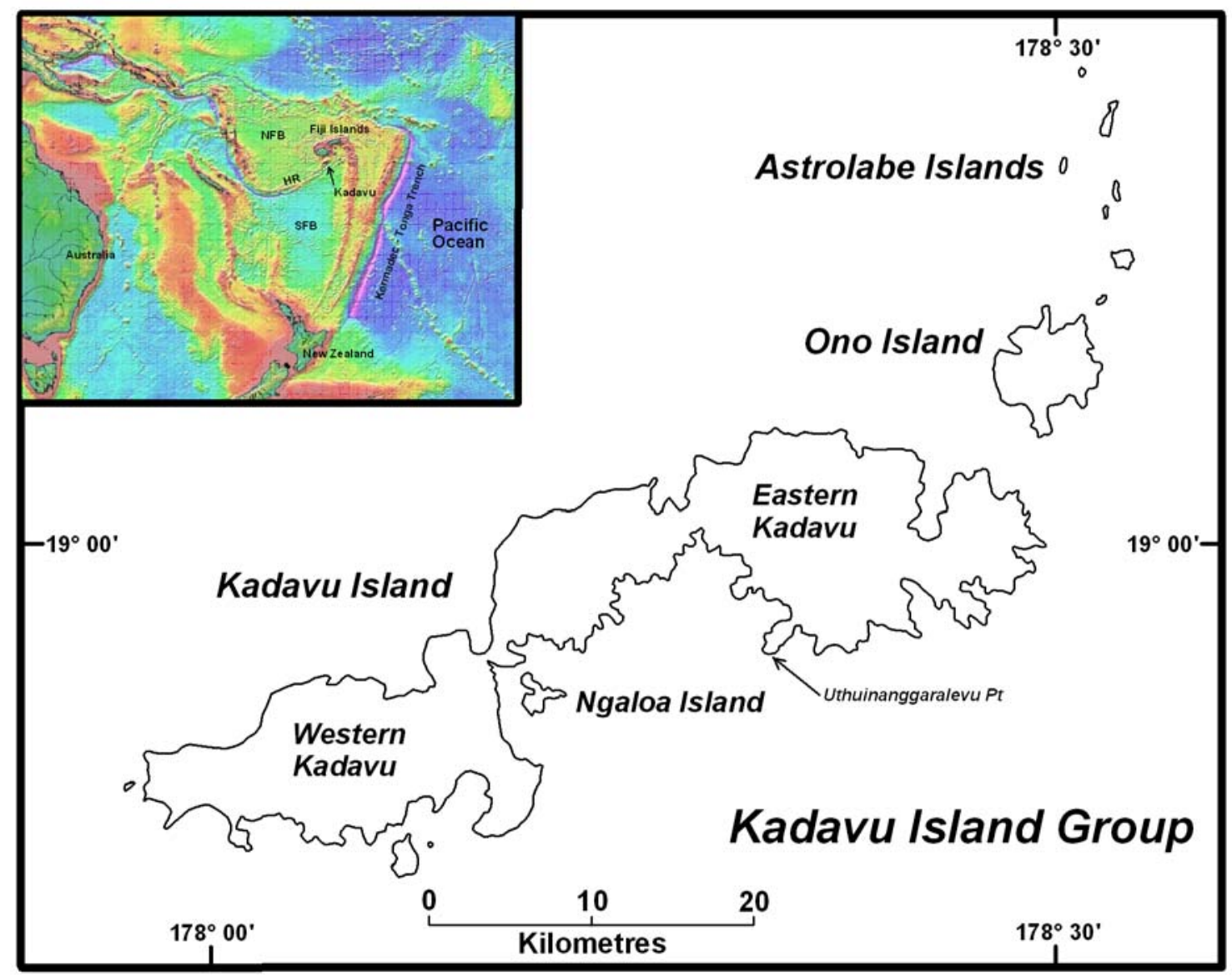



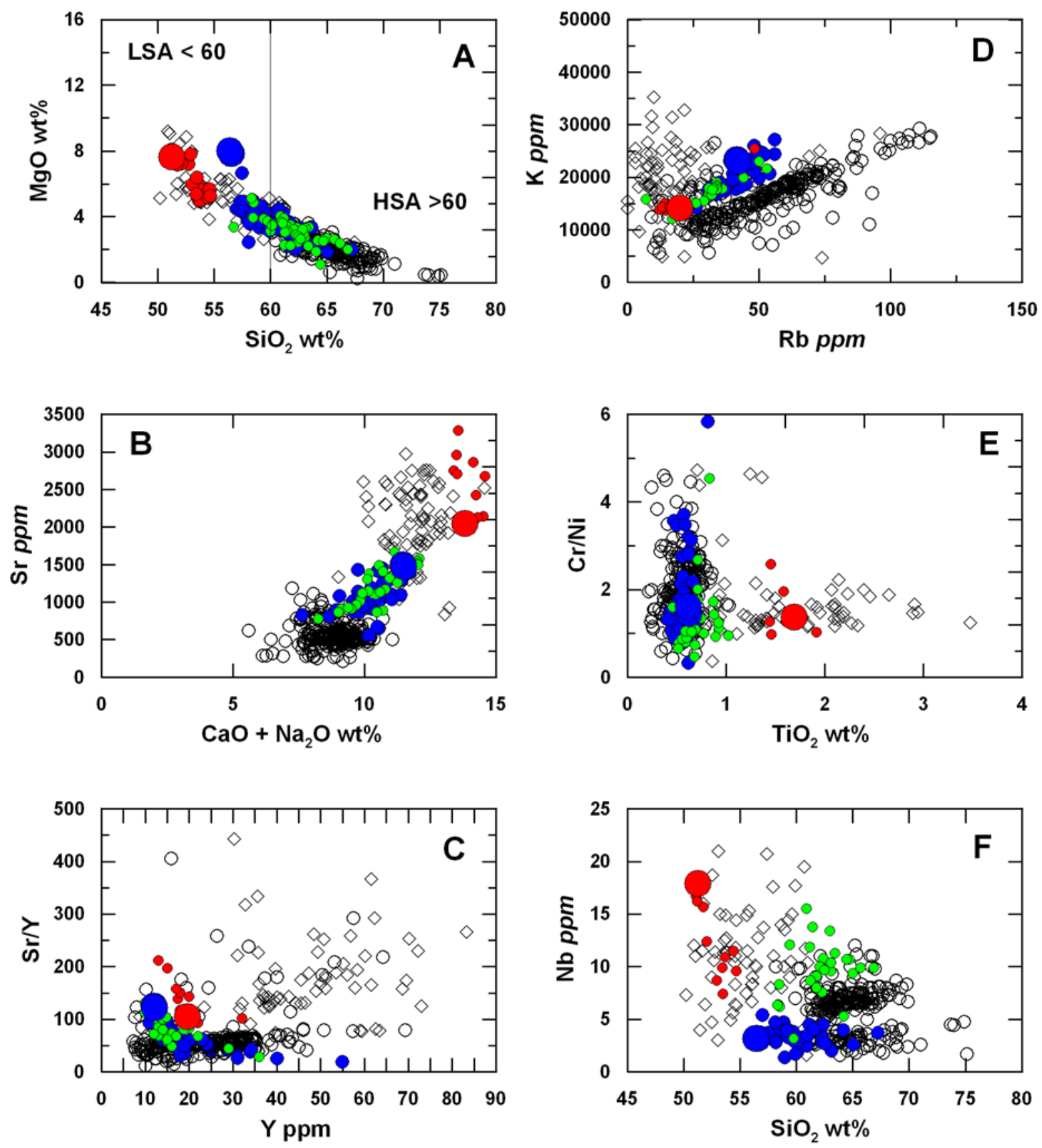

East Kadavu high magnesian adakite
East Kadavu adakites
Ngaloa high magnesian adakite
Ngaloa adakites
Western Kadavu adakites
$\diamond$ High $\mathrm{SiO}_{2}$ adakites
$\mathrm{SiO}_{2}$ adakites




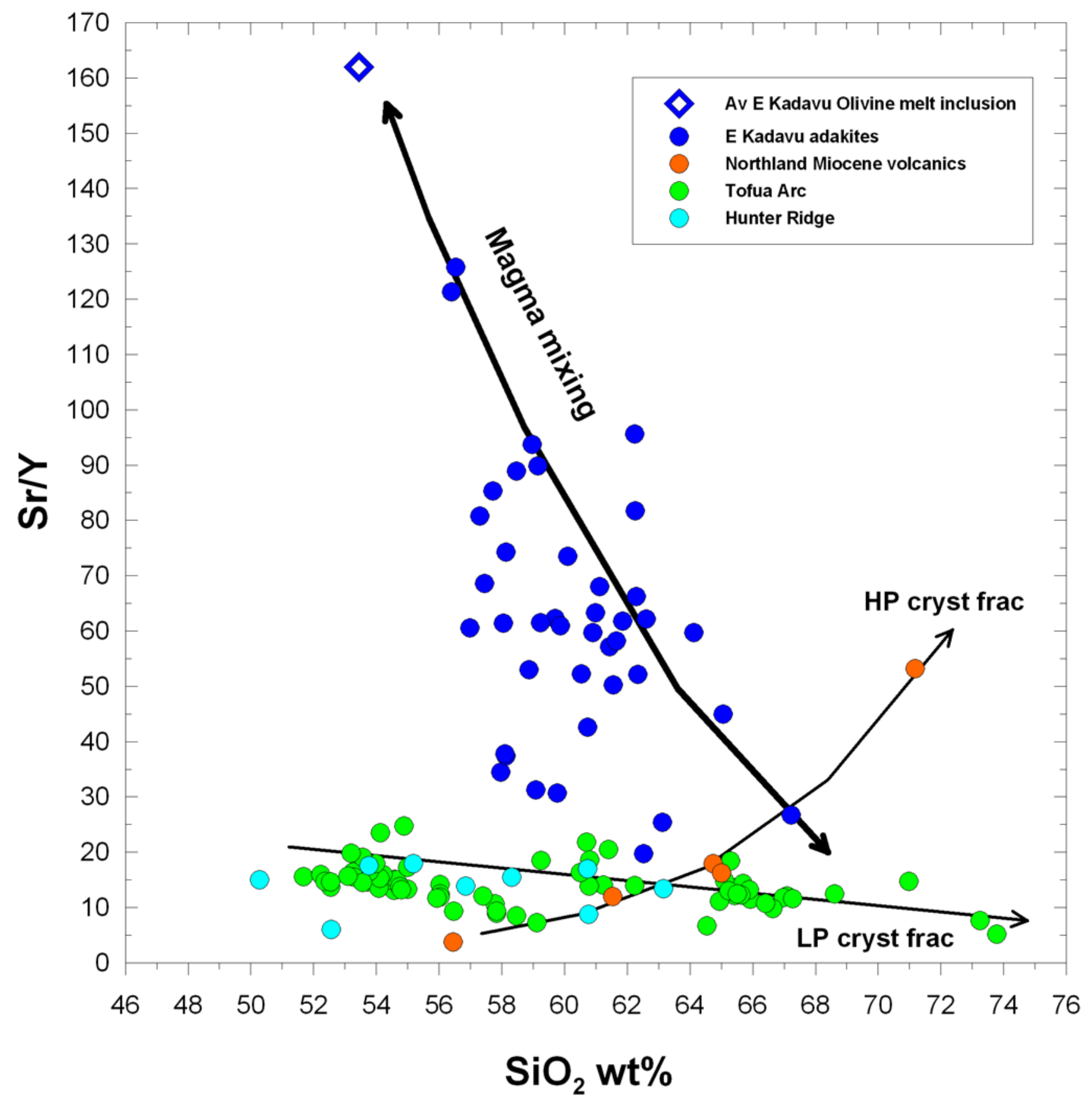




\begin{tabular}{|c|c|c|c|c|c|c|c|}
\hline Sample no. & Description & Lat & Long & $\mathrm{SiO2}$ & TiO2 & $\mathrm{Al2O3}$ & FeOT \\
\hline AV37 & lava flow & 1904.2 & 17803.9 & 60.85 & 0.87 & 15.96 & 4.41 \\
\hline AV84 & lava flow & 1909.3 & 17810.3 & 58.43 & 0.83 & 17.13 & 5.97 \\
\hline AV54 & lava flow & 1908.9 & 17801.2 & 59.37 & 1.02 & 16.57 & 4.73 \\
\hline AV70 & lava flow & 1910.8 & 17806.7 & 61.16 & 0.93 & 16.67 & 4.43 \\
\hline AV78 & lava flow & 1908.3 & 17811.2 & 59.74 & 0.52 & 17.73 & 5.63 \\
\hline AV76 & float in creek & 1907.1 & 17808.4 & 61.80 & 0.69 & 16.67 & 4.60 \\
\hline AV77 & lava flow & 1908.0 & 17807.3 & 61.42 & 0.92 & 16.19 & 4.59 \\
\hline AV48 & lava flow & 1908.9 & 17758.4 & 63.39 & 0.89 & 15.84 & 3.69 \\
\hline AV72 & lava flow & 1907.8 & 17804.8 & 62.37 & 0.77 & 16.50 & 4.42 \\
\hline AV62 & lava flow & 1908 & 17803.2 & 62.04 & 0.63 & 16.97 & 4.18 \\
\hline AV73 & lava flow & 1906.2 & 17804.2 & 63.06 & 0.68 & 16.98 & 3.93 \\
\hline AV85 & lava flow & 1907.9 & 17809.3 & 62.93 & 0.74 & 16.58 & 3.97 \\
\hline AV91 & lava flow & 1907.0 & 17811.3 & 62.26 & 0.59 & 17.35 & 4.56 \\
\hline AV94 & lava flow & 1904.0 & 17809.4 & 63.05 & 0.64 & 17.18 & 4.09 \\
\hline AV14 & lava flow & 1906.7 & 17758.1 & 64.98 & 0.55 & 16.35 & 3.28 \\
\hline AV50 & float in creek & 1907.8 & 17759.0 & 65.66 & 0.60 & 16.18 & 3.35 \\
\hline AV40 & float in creek & 1905.3 & 17801.7 & 64.17 & 0.52 & 17.69 & 3.39 \\
\hline AV5 & lava flow & 1902.7 & 17808.8 & 64.69 & 0.58 & 16.76 & 3.48 \\
\hline AV66 & float on beach & 1909.7 & 17803.8 & 62.46 & 0.68 & 17.69 & 3.94 \\
\hline AV57 & lava flow & 1908.7 & 17802.5 & 61.21 & 0.88 & 17.68 & 4.51 \\
\hline AV59 & lava flow & 1908.3 & 17802.4 & 61.76 & 0.65 & 18.36 & 4.02 \\
\hline AV90 & lava flow & 1907.0 & 17811.4 & 66.87 & 0.46 & 16.18 & 3.16 \\
\hline AV31 & clast in breccia & 1904.9 & 17807.0 & 64.44 & 0.70 & 17.57 & 4.27 \\
\hline ST1 & lava flow & 1907.8 & 17758.4 & 66.17 & 0.48 & 16.54 & 2.94 \\
\hline ST5 & lava flow & 1906.7 & 17757.8 & 60.94 & 0.96 & 16.36 & 4.33 \\
\hline ST14 & lava flow & 1906.4 & 17759.2 & 63.10 & 0.75 & 16.66 & 3.39 \\
\hline ST16 & float on beach & 1904.5 & 17802.5 & 64.05 & 0.52 & 17.82 & 3.60 \\
\hline ST17 & float on beach & 1904.5 & 17802.5 & 62.95 & 0.58 & 17.60 & 3.71 \\
\hline ST18 & float on beach & 1904.5 & 17802.5 & 62.88 & 0.61 & 17.23 & 3.79 \\
\hline ST19 & float on beach & 1904.5 & 17802.5 & 62.44 & 0.72 & 17.58 & 3.88 \\
\hline ST20 & lava flow & 1900.2 & 17801.8 & 63.32 & 0.81 & 16.45 & 4.19 \\
\hline ST21 & lava flow & 1900.2 & 17801.8 & 61.50 & 0.65 & 18.19 & 3.77 \\
\hline ST22 & lava flow & 1909.5 & 17809.5 & 61.27 & 0.49 & 17.54 & 5.01 \\
\hline ST23 & clast in breccia & 1909.5 & 17809.5 & 60.17 & 0.54 & 17.77 & 5.14 \\
\hline ST24 & clast in breccia & 1909.5 & 17809.5 & 59.97 & 0.55 & 18.40 & 5.14 \\
\hline ST25 & float on beach & 1909.5 & 17808.8 & 61.09 & 0.50 & 17.35 & 4.94 \\
\hline ST27 & float on beach & 1909.5 & 17808.8 & 58.49 & 0.71 & 15.55 & 5.57 \\
\hline ST28 & float on beach & 1909.5 & 17808.8 & 60.15 & 0.68 & 17.46 & 5.22 \\
\hline ST29 & float on beach & 1909.5 & 17808.8 & 62.68 & 0.49 & 17.36 & 4.51 \\
\hline ST31 & float on beach & 1909.5 & 17808.8 & 58.32 & 0.71 & 15.43 & 5.58 \\
\hline
\end{tabular}




\begin{tabular}{|c|c|c|c|c|c|c|c|c|}
\hline MnO & MgO & $\mathrm{CaO}$ & $\mathrm{Na2O}$ & $\mathrm{K} 2 \mathrm{O}$ & P2O5 & LOI & Sc & V \\
\hline 0.08 & 4.06 & 6.34 & 4.62 & 2.30 & 0.51 & 0.45 & 14 & 129 \\
\hline 0.09 & 3.93 & 6.96 & 4.29 & 1.90 & 0.46 & 1.86 & 19 & 198 \\
\hline 0.08 & 3.87 & 7.26 & 4.82 & 1.72 & 0.56 & 0.96 & 14 & 158 \\
\hline 0.07 & 3.91 & 5.89 & 4.66 & 1.81 & 0.46 & 2.91 & 11 & 130 \\
\hline 0.09 & 3.75 & 6.57 & 4.13 & 1.60 & 0.23 & 1.28 & 20 & 178 \\
\hline 0.08 & 3.50 & 5.89 & 4.53 & 1.94 & 0.30 & 0.71 & 14 & 139 \\
\hline 0.08 & 3.46 & 6.04 & 4.75 & 2.06 & 0.49 & 0.83 & 13 & 144 \\
\hline 0.07 & 3.37 & 5.56 & 4.61 & 2.03 & 0.55 & 1.34 & 9 & 108 \\
\hline 0.08 & 3.34 & 5.56 & 4.59 & 2.03 & 0.34 & 0.81 & 14 & 136 \\
\hline 0.07 & 3.22 & 6.07 & 4.39 & 2.15 & 0.27 & 1.20 & 14 & 122 \\
\hline 0.06 & 3.23 & 4.99 & 4.87 & 1.86 & 0.33 & 0.76 & 12 & 116 \\
\hline 0.05 & 3.12 & 4.97 & 4.87 & 2.40 & 0.36 & 2.31 & 13 & 123 \\
\hline 0.08 & 2.89 & 5.52 & 4.59 & 1.82 & 0.33 & 1.54 & 13 & 139 \\
\hline 0.07 & 2.75 & 5.18 & 4.51 & 2.23 & 0.30 & 1.65 & 11 & 108 \\
\hline 0.07 & 2.70 & 4.44 & 5.05 & 2.19 & 0.38 & 1.42 & 8 & 78 \\
\hline 0.07 & 2.63 & 4.22 & 4.89 & 2.00 & 0.39 & 1.71 & 11 & 94 \\
\hline 0.05 & 2.56 & 4.84 & 5.09 & 1.46 & 0.23 & 1.49 & 10 & 97 \\
\hline 0.07 & 2.52 & 4.49 & 4.51 & 2.61 & 0.28 & 2.06 & 11 & 90 \\
\hline 0.07 & 2.46 & 5.38 & 5.23 & 1.71 & 0.37 & 1.16 & 9 & 111 \\
\hline 0.07 & 2.27 & 6.26 & 4.88 & 1.78 & 0.46 & 1.28 & 11 & 133 \\
\hline 0.05 & 2.26 & 5.36 & 5.35 & 1.82 & 0.38 & 0.91 & 10 & 105 \\
\hline 0.06 & 2.02 & 3.94 & 4.30 & 2.77 & 0.24 & 2.49 & 9 & 70 \\
\hline 0.08 & 1.10 & 4.30 & 5.08 & 2.13 & 0.33 & 1.86 & 15 & 115 \\
\hline 0.06 & 2.40 & 4.32 & 4.86 & 1.90 & 0.33 & 0.95 & & \\
\hline 0.08 & 3.68 & 6.34 & 5.01 & 1.81 & 0.49 & 0.94 & & \\
\hline 0.06 & 3.30 & 5.55 & 4.79 & 1.97 & 0.44 & 1.27 & & \\
\hline 0.04 & 1.88 & 5.22 & 5.16 & 1.47 & 0.24 & 0.8 & & \\
\hline 0.06 & 2.92 & 5.49 & 4.73 & 1.66 & 0.30 & 1.56 & & \\
\hline 0.07 & 3.26 & 5.35 & 4.86 & 1.65 & 0.30 & 0.53 & & \\
\hline 0.07 & 2.80 & 5.48 & 4.94 & 1.72 & 0.37 & 1.52 & & \\
\hline 0.04 & 2.21 & 5.25 & 4.75 & 2.58 & 0.38 & 3.1 & & \\
\hline 0.06 & 3.15 & 5.46 & 4.92 & 1.92 & 0.38 & 3.63 & & \\
\hline 0.06 & 3.08 & 5.89 & 4.82 & 1.63 & 0.22 & 1.12 & & \\
\hline 0.08 & 3.55 & 6.48 & 4.24 & 1.76 & 0.27 & 2.53 & & \\
\hline 0.08 & 3.13 & 6.26 & 4.66 & 1.55 & 0.24 & 4.08 & & \\
\hline 0.08 & 3.60 & 6.15 & 4.27 & 1.80 & 0.23 & 1.6 & & \\
\hline 0.12 & 4.86 & 7.61 & 4.39 & 2.17 & 0.53 & 0.93 & 19 & 183 \\
\hline 0.08 & 3.47 & 6.34 & 4.45 & 1.78 & 0.36 & 1.1 & & \\
\hline 0.08 & 2.67 & 5.39 & 4.46 & 2.10 & 0.25 & 1 & & \\
\hline 0.15 & 5.13 & 7.71 & 4.32 & 2.13 & 0.52 & 0.91 & 19 & 182 \\
\hline
\end{tabular}




\begin{tabular}{rrrrrrrrr}
$\mathbf{C r}$ & $\mathbf{N i}$ & $\mathbf{C u}$ & $\mathbf{Z n}$ & $\mathbf{R b}$ & $\mathbf{S r}$ & $\mathbf{Y}$ & $\mathbf{Z r}$ & $\mathbf{N b}$ \\
\hline 97 & 56 & & & 34 & 1317 & 10 & 204 & 15.5 \\
59 & 13 & & & 7 & 1263 & 13 & 176 & 8.3 \\
109 & 114 & 44 & 57 & 23 & 1580 & 13 & 174 & 12.1 \\
54 & 48 & & & 25 & 1488 & 13 & 191 & 11.9 \\
15 & 19 & & & 20 & 888 & 13 & 102 & 3.2 \\
24 & 22 & & & 30 & 1102 & 13 & 132 & 9.1 \\
56 & 45 & & & 31 & 1171 & 16 & 182 & 13.8 \\
51 & 55 & 41 & 51 & 31 & 1377 & 14 & 172 & 11.3 \\
27 & 27 & & & 32 & 1151 & 17 & 151 & 10.8 \\
29 & 25 & & & 36 & 870 & 15 & 162 & 10.0 \\
34 & 46 & & & 29 & 1048 & 14 & 150 & 9.6 \\
39 & 29 & & & 44 & 1015 & 36 & 164 & 13.4 \\
12 & 15 & & & 25 & 1304 & 29 & 134 & 7.6 \\
18 & 18 & & & 34 & 961 & 14 & 175 & 10.4 \\
21 & 24 & & & 34 & 909 & 13 & 147 & 9.4 \\
34 & 38 & & & 32 & 914 & 13 & 180 & 9.9 \\
33 & 50 & & & 17 & 1120 & 10 & 129 & 5.3 \\
20 & 19 & & & 53 & 867 & 12 & 171 & 10.7 \\
8 & 17 & & & 23 & 1136 & 14 & 161 & 9.7 \\
46 & 32 & 63 & \multirow{2}{*}{60} & 23 & 1667 & 20 & 178 & 8.7 \\
29 & 28 & & & 26 & 1411 & 14 & 171 & 8.0 \\
16 & 10 & 34 & \multirow{2}{*}{38} & 50 & 779 & 16 & 185 & 9.9 \\
24 & 18 & & & 34 & 927 & 15 & 152 & 10.7
\end{tabular}

$\begin{array}{rrrrrrrrr}140 & 52 & 39 & 62 & 31 & 1512 & 19 & 182 & 6.2 \\ 139 & 79 & 44 & 99 & 32 & 1497 & 22 & 180 & 6.4\end{array}$




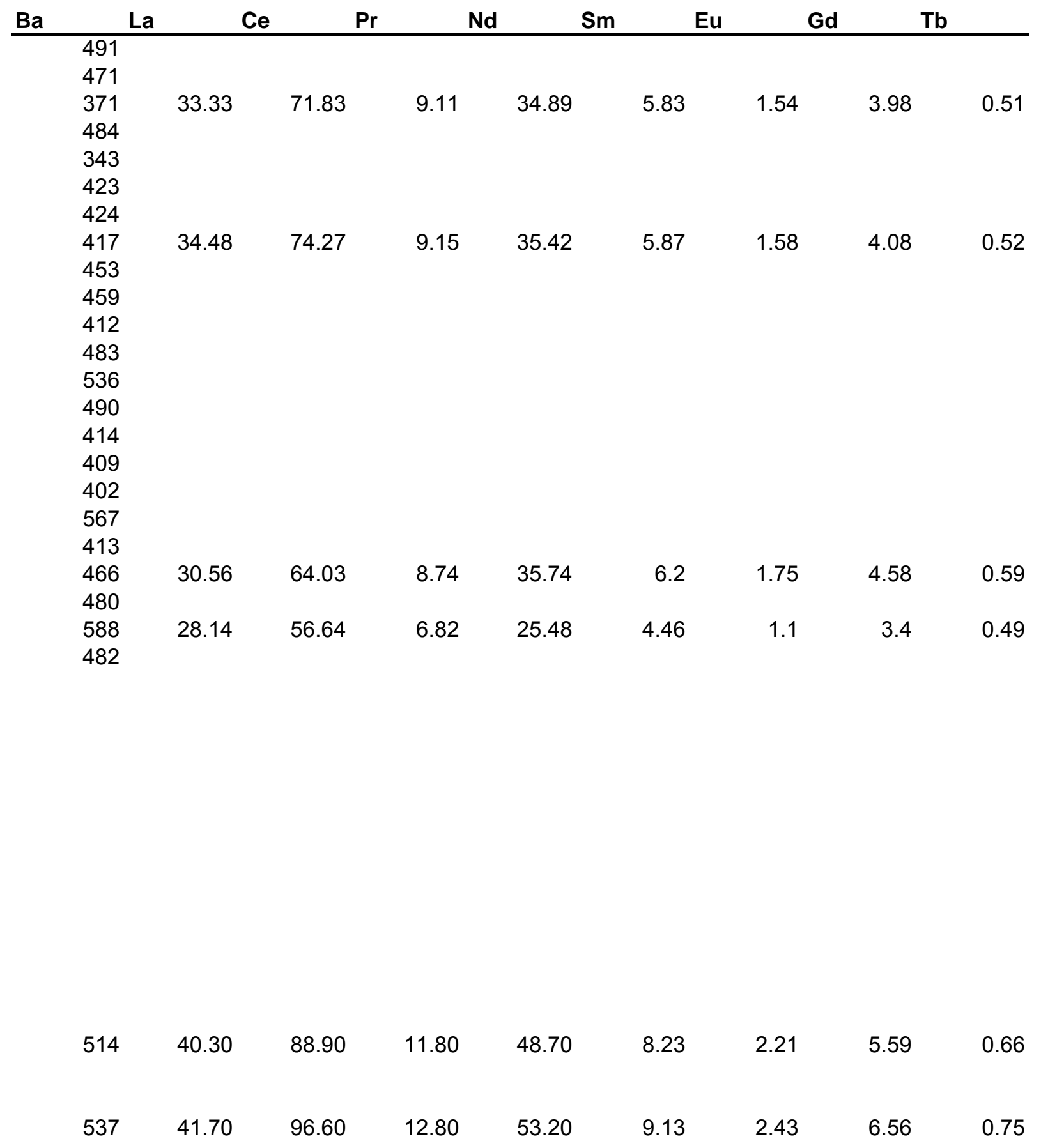


DR2008118

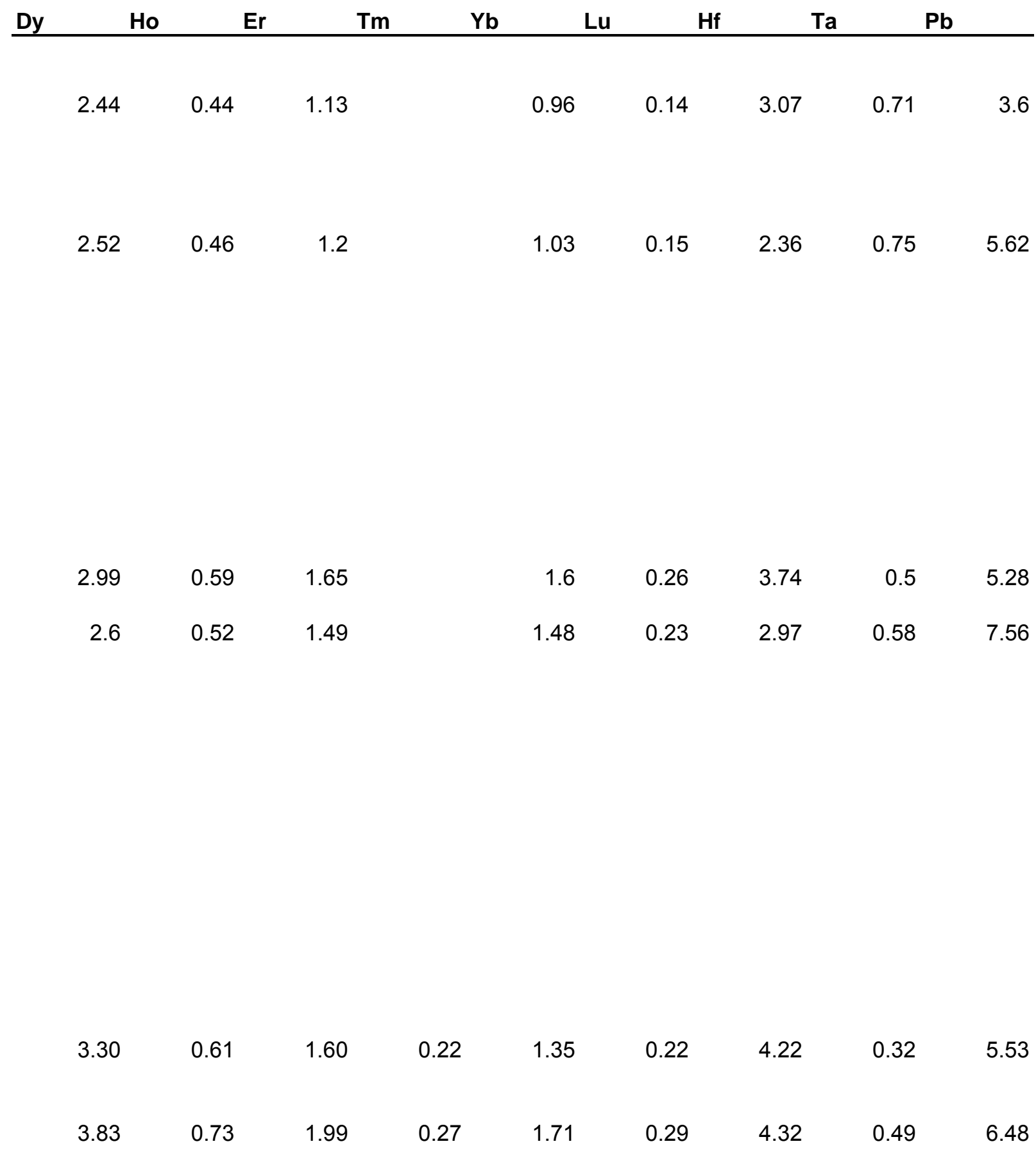


DR2008118

Th

U

$3.94 \quad 1.05$

$5.44 \quad 1.91$

$\begin{array}{ll}3.8 & 1.2\end{array}$

$5.36 \quad 1.82$

$4.68 \quad 1.41$

$4.61 \quad 1.46$ 